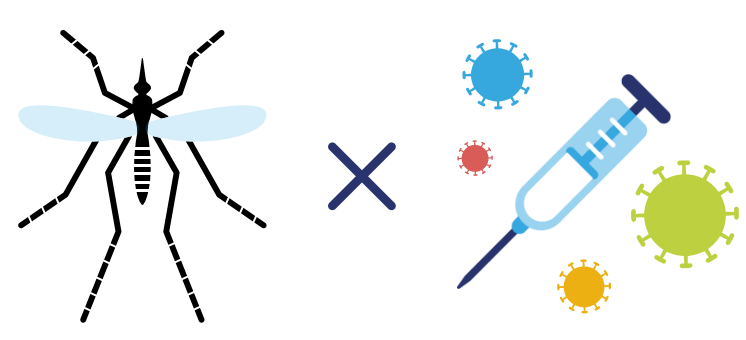

\title{
ANÁLISE CIENTÍFICA E TECNOLÓGICA SOBRE DENGUE E H1N1: COMPARATIVO ENTRE DOENÇA TROPICAL NEGLIGENCIADA E NÃO NEGLIGENCIADA
}

\author{
SCIENTIFIC AND TECHNOLOGICAL ANALYSIS OF DENGUE AND H1N1: COMPARISON
}

BETWEEN A NEGLECTED TROPICAL DISEASE AND A NON-NEGLECTED DISEASE

\section{Ana Cintia Ribeiro da Silva}

Bacharel em Enfermagem, Mestre em Inovação Tecnológica pela Universidade Federal do Triângulo Mineiro - UFTM, anacintiaribeiros@gmail.com

Maira Silveira de Almeida

Bibliotecária Documentalista, Mestre em Inovação Tecnológica pela Universidade Federal do Triângulo Mineiro - UFTM, mairajulio@gmail.com

Maria Angélica Santa Cruz Nogueira Favaro

Bacharel em Medicina, Médica Infectologista pela Universidade Federal do Triangulo Mineiro,

angelicanogueira@hotmail.com

Geoffroy Roger Pointer Malpass

Professor do Departamento de Engenharia Química e do Mestrado Profissional em Inovação Tecnológica da Universidade Federal do Triangulo Mineiro, geoffroy.malpass@uftm.edu.br

Ana Claudia Granato

Professora do Departamento de Engenharia Química e do Mestrado Profissional em Inovação Tecnológica da Universidade Federal do Triangulo Mineiro, ana.malpass@uftm.edu.br

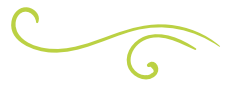

\section{RESUMO}

O avanço tecnológico é uma importante estratégia para o desenvolvimento econômico e social mundial. Dessa forma, o estudo realizou uma análise comparativa entre documentos de patentes sobre dengue e H1N1, no período de 2001 a 2016, utilizando como fonte de informação o banco de dados Derwent Innovations Index. Para tanto, analisou os seguintes fatores: distribuição geográfica dos depósitos; tipologia; Classificação Internacional das Patentes (CIP); foco tecnológico e evolução temporal dos pedidos de patentes no período da pesquisa. Ao utilizar a expressão "dengue", encontrou 2660 famílias de patentes, enquanto que, para "H1N1", foram 1103 famílias. Ao verificar a distribuição geográfica, observou domínio dos Estados Unidos em ambas as patologias. $\mathrm{Na}$ categoria dos depositantes das patentes, houve destaque para "pessoa física". Já para a tendência temática das inovações, a partir da CIP, verificou a predominância da seção "Necessidades Humanas", com "Ciência Médica ou Veterinária e Higiene" no nível de classe; e para subclasse, as mais predominantes foram "Atividade terapêutica 
específica de compostos químicos ou preparações medicinais" e "Preparações para finalidades médicas, odontológicas ou higiênicas". Na evolução temporal anual, observou que a média para dengue foi de 177 patentes ao ano, com incremento de 22,43 e aceleração negativa, após 2007, de 1,40 anualmente. Para H1N1, a média anual foi de 88,57 patentes, incremento de 21,19 e aceleração negativa de 1,02 ao ano. $\bigcirc$ monitoramento por meio da análise do registro de patentes é de fundamental importância para a tomada de decisões que geram impacto na situação da saúde pública mundial, tais como: o direito à saúde e o acesso aos serviços, em especial, nos países em desenvolvimento.

Palavras-chave: Patente. Doença tropical negligenciada. Dengue. H1N1. Derwent innovations index.

\section{ABSTRACT}

Technological advancement is an important strategy for world economic and social development. In this context, innovation is highlighted and directly linked to the number of patent deposits for the pathologies dengue and influenza $A(H 1 N 1)$. A comparison between the two diseases, one considered a neglected tropical disease and the other not, in the period from 2001 to 2016, was performed using the Derwent Innovations Index database as the source of information. The factors analyzed were geographic distribution of the registrations; the typology (depositor); the description of the applications according to the International Patent Classification (IPC); the technological focus and the temporal evolution of patent applications in the period of the study. When using the term "dengue", 2660 patent families were found, while "H1N1" found 1103 families. When verifying the geographical distribution, domination of the United States in both pathologies was verified. For the thematic trend of innovations, from the IPC, the predominance was found for the section "Human Needs", with "Medical or Veterinary Science and Hygiene" at the class level and for the subclass, the most predominant were "Therapeutic activity specific for chemical compounds or medical preparations" and "Preparations for medical, dental or hygienic purposes". Analyzing the annual time evolution, it is observed that the average for dengue was 177 patents per year, with an increase of 22.43 and a negative acceleration, after 2007, of 1.40 annually. And for $\mathrm{H} 1 \mathrm{~N} 1$, the annual average was 88.57 patents, an increase of 21.19 and a negative acceleration of 1.02 a year. Monitoring by means of the analysis of the patent registry is a fundamental contribution to making decisions that have an impact on the world public health situation, such as the right to health and access to services, especially in developing countries.

Keywords: Patent. Tropical neglected diseases. Dengue. H1N1. Derwent Innovations Index

\section{INTRODUÇÃO}

O desempenho de um país no que se refere à inovação, bem como o seu desenvolvimento tecnológico, pode ser avaliado pelo número de pedidos de patentes existentes (MENDES et al., 2013), além de ser impulsionado ao gerir políticas públicas consistentes em propriedade intelectual (PEREIRA, 2011). Os documentos de patentes contêm informações detalhadas dos processos e invenções e são utilizados para verificação da tecnologia e do monitoramento prospectivo, com intuito de desencadear processos de construção para um futuro desejável (TEIXEIRA; SOUZA, 2013).

As ações inovadoras deste estudo serão examinadas sob a ótica da doença tropical negligenciada (DTN) dengue e da doença não negligenciada H1N1, estabelecendo um comparativo entre as patentes depositadas. 
O próprio significado da palavra negligenciada já indica baixa prioridade. Nos países desenvolvidos, as DTNs são quase inexistentes e esses espaços são detentores de um grande número de patentes (RODRIGUES, 2013). A dengue é considerada a doença viral mais ocorrente e elencada como um dos principais problemas da saúde pública mundial (LINDOSO; LINDOSO, 2009). Já a infecção pelo vírus influenza A (H1N1), doença não negligenciada respiratória aguda, de origem suína, também é considerada causadora de grande preocupação para a saúde pública mundial (ROSSETTO; LUNA, 2016). Diante da magnitude e da gravidade da dengue e da influenza A H1N1, bem como das dificuldades encontradas para controlá-las, tais patologias foram selecionadas como objetos deste estudo.

Os objetivos desta pesquisa se relacionam à análise dos indicadores científicos e tecnológicos dos depósitos de patentes mundiais referentes à dengue, doença tropical negligenciada e à influenza A H1N1, doença não negligenciada, no período de 2001 a 2016. Especificamente, pretende-se determinar a distribuição geográfica dos países depositantes das patentes; identificar a tipologia dos depositantes; verificar a classificação predominante das patentes conforme a Classificação Internacional de Patentes - CIP; descrever o foco tecnológico e analisar a evolução temporal dos depósitos das patentes no período de 2001 a 2016.

A escolha da pesquisa justifica-se por ser um tema relevante e de interesse para a saúde pública. Tanto a dengue quanto a influenza A H1N1 são virais, sendo uma considerada negligenciada e a outra não, respectivamente. $\bigcirc$ levantamento das patentes possibilitará um estudo comparativo, no intuito de ter uma visão mais ampla das ações e pesquisas realizadas no mundo para essas doenças. Por ser um problema de saúde pública mundial, o mapeamento e os estudos inovadores contribuem para um melhor planejamento de ações e melhorias da saúde. A inovação tecnológica trazida pelas patentes é essencial para o desenvolvimento em saúde de um país (TEIXEIRA; SOUZA, 2013).

\section{VISÃO GERAL DAS DOENÇAS NEGLIGENCIADAS}

As DTNs são patologias que atingem principalmente populações de baixa renda que, em sua maioria, vivem em países em desenvolvimento, tais como África, Ásia e América Latina (DESSOY et al., 2013). Diversas doenças são consideradas negligenciadas, entre elas, dengue, febre amarela, esquistossomose, leishmaniose, helmintíases, raiva, tripanossomíase humana africana, doença de Chagas, malária, hanseníase, sífilis, entre outras (SOUZA, 2010).

Dessoy et al. (2013) apontam que a presença das DTNs está relacionada a falhas de mercado e de políticas públicas agravadas pelo inadequado investimento em inovação e programas de pesquisa e desenvolvimento. Morel (2006) corrobora exemplificando as possíveis causas como sendo: o conhecimento insuficiente que exige mais pesquisas; questões de mercado, com produtos de preços abusivos; assim como a saúde pública que, por sua vez, apresenta planejamento deficiente não utilizando diversos medicamentos ou métodos de controle das doenças. Ainda, segundo Feres e Silva (2016), ações direcionadas ao saneamento básico são as que mais contribuem para a redução do acometimento das DTNs na população, entretanto, a descoberta de novos produtos, a inovação e o patenteamento também têm um papel efetivo.

Nas regiões tropicais e subtropicais, estão os países com menor Índice de Desenvolvimento Humano (IDH) e maior número de portadores de DTNs, relacionando, dessa forma, a pobreza e a ocorrência dessas patologias. $\bigcirc$ Brasil ocupa a $70^{a}$ posição no IDH e concentra um número significativo de DTNs. As principais 
DTNs no Brasil são: dengue, doença de Chagas, hanseníase, malária, esquistossomose, tuberculose, leishmaniose visceral e cutânea (LINDOSO, 2014).

\section{DENGUE}

Considerada a doença viral mais ocorrente, a dengue é uma arbovirose responsável por um grave problema de saúde pública no mundo, é infecciosa e não contagiosa (DALBEM et al., 2014; LINDOSO; LINDOSO, 2009). Arboviroses são doenças virais, transmitidas por vetores e possuem alta incidência e dispersão, sendo o arbovírus do gênero Flavivírus, pertencente à família Flaviviridae (MANIERO et al., 2016). $O$ aumento da dengue, em escala mundial, deve-se à urbanização da sociedade. No século $X X$, a dengue foi quase erradicada, entretanto, as estratégias de controle foram insuficientes. Houve o ressurgimento das populações do Aedes em toda a América e aumento da disseminação global (SHARP et al., 2017).

Existem quatro sorotipos conhecidos do vírus da dengue: DEN-1, DEN-2, DEN-3 e DEN-4, que estão espalhados nas regiões tropicais e subtropicais de todo o mundo (CARVALHO, 2016; SHARP et al., 2017; DALBEM et al., 2014). O vírus é inoculado na pessoa sadia devido à picada da fêmea do Aedes aegypti, que atinge as células, realiza a replicação e leva à viremia. A produção de anticorpos IGM inicia-se entre o quinto e o sexto dia. Possui alta taxa de morbidade e a pessoa infectada pode apresentar-se de forma assintomática ou com sintomas clínicos que variam desde o estado febril até a forma mais grave da doença. Pode apresentar-se como dengue clássica, dengue com sinais de alarme e dengue grave (DIAS et al., 2010).

O diagnóstico clínico precoce aumenta as chances de sobrevida dos pacientes, já o diagnóstico laboratorial é feito pela detecção de antígenos virais (BRASIL, 2017a;
MANIERO et al., 2016). Conforme estudo realizado por Sharp et al. (2017), mesmo sendo endêmica em diversas áreas, o sub-reconhecimento dos casos de dengue prejudica o controle dos vetores. Valle et al. (2016) apontam que as deficiências para controle e prevenção estão além do quesito saúde, considerando que fatores socioeconômicos e ambientais não podem ser ignorados em detrimento de intervenções somente biomédicas ou tecnológicas.

Estudo realizado por Feres e Silva (2016) verificou a negligência no quesito inventividade quando são comparadas as doenças negligenciadas com outras enfermidades que possuem menor carga global, mas, ainda assim, são mais bem amparadas pela cadeia inventiva. Conhecer a epidemiologia da dengue é essencial para o desenvolvimento de novas práticas para controle, prevenção, aumento da consciência clínica e melhoria na vigilância e no diagnóstico (SHARP et al., 2017). Os estudos em busca de extratos e substâncias naturais que não sejam tóxicas para o ambiente e combatam o vetor de forma eficiente estão em ascendência (PEREIRA et al., 2014).

\section{INFLUENZA A H1N1}

A influenza, também conhecida como gripe, é uma infecção respiratória aguda, febril e autolimitada, causada por vírus que circulam por todo o mundo, podendo causar epidemias e até pandemias $(\mathrm{WHO}$, 2017a; BRASIL, 2017b). Dependendo do perfil antigênico, os vírus influenza podem ser divididos em $A, B$ e $C$, sendo extremamente mutáveis (PAULA; RIBAS, 2015). O tipo A é responsável por diversas pandemias e possui os subtipos H1N1 e o H3N2, que atualmente são circulantes nos seres humanos. $\mathrm{O}$ vírus H1N1 é sazonal, ocorre principalmente nos períodos mais frios e gerou a pandemia de 2009 (BRASIL, 2017b). Entre os desafios para controlar as doenças infecciosas globais está o desenvolvimento de 
novos fármacos e vacinas. É uma preocupação constante para a saúde pública mundial, portanto, a OMS trabalha fortalecendo todas as regiões do globo, estimulando o desenvolvimento de métodos diagnósticos, a verificação de susceptibilidade, respostas aos surtos e aumento da cobertura vacinal (WHO, 2017b).

\section{PATENTE}

Patente é um documento fornecido ao inventor ou a quem a solicitou, que oferece direitos sobre a invenção. No Brasil, ela é concedida pelo Instituto Nacional de Propriedade Industrial (INPI) (PERUCCHI; MUELLER, 2014b). É um privilégio concedido pelo Estado aos inventores ou mesmo aos detentores do direito para explorar um invento (TEIXEIRA; SOUZA, 2013). Ao conceder a patente ao inventor, o Estado garante o monopólio do invento por um período de tempo. Após esse período, todo o conteúdo passa a ser de domínio público (TEIXEIRA; SOUZA 2013). As patentes são inovações protegidas legalmente porque possuem valor comercial. No Brasil, as leis que tratam da Inovação são: Lei da Inovação Tecnológica $n^{\circ}$ 10.973, de 2004 e a Lei de Propriedade Industrial $n^{\circ}$ 9.279, de 14 de maio de 1996 (PERUCCHI; MUELLER, 2014a).

Um termo muito utilizado é o documento de patente, que está relacionado tanto aos pedidos de patentes depositados quanto às patentes já concedidas após toda a tramitação. Esse documento de patente inclui na sua estrutura: folha de rosto, que traz informações bibliográficas; relatório descritivo; reivindicações; desenhos, quando houver; e resumo (TEIXEIRA; SOUZA, 2013). Conforme Muller e Perucchi (2014b), o depósito não significa concessão e a concessão não significa que ela será explorada de alguma maneira. Um documento de patente descreve informações detalhadas de tecnologias e processos que só podem ser encontrados nesse tipo de documento, focando, portanto, na prospecção tecnológica (MUELLER; PERUCCHI, 2014b). "Nem toda tecnologia é patenteada pela empresa que a desenvolve. Algumas são mantidas como segredo industrial" (TEIXEIRA; SOUZA, 2013, p. 8).

No Brasil, a Lei da Propriedade Industrial (LPI nº 9279 de 14 de maio de 1996) prevê dois tipos de patentes: a de invenção (PI) e de modelo de utilidade (MU) (BRASIL, 1996). Com o objetivo de estimular a geração de novas patentes, foram criadas, mais recentemente, a Lei da Inovação (LI n 10.973 de 2004) e a Lei do Bem ( $n^{\circ} 11.196$ de 2005) (LAMANA; KOVALESKI, 2010). O INPI é o responsável pela concessão da propriedade intelectual no Brasil e foi criado em 1970 (LUNA; et al., 2007). A patente evita a duplicidade de esforços em pesquisas (TEIXEIRA; SOUZA, 2013), por sua vez, a criação intelectual protegida contribui para o desenvolvimento econômico e tecnológico de um país (INPI, 2015).

Calmanovici (2011) aponta que a atividade inovadora é gerada, principalmente, pelo fator competitividade, motivado pela demanda de mercado, ocasionando impacto e consequências para toda a sociedade. Um documento de patente, que possui as mais inovadoras tecnologias, é considerado privilegiado por ser confiável, padronizado e abrangente, além de antecipar as futuras tendências industriais, relacionando tecnologias para diversificados fins (TEIXEIRA; SOUZA, 2013). Os documentos de patentes incluem diversos campos tecnológicos. Portanto, para facilitar o acesso, são classificados de acordo com a CIP (TEIXEIRA; SOUZA, 2013). A Organização Mundial de Propriedade Intelectual (OMPI) administra os direitos de propriedade intelectual no mundo e gera a CIP, mantendo sempre os códigos atualizados conforme a evolução da tecnologia (ANTUNES et al., 2012). Um documento de patente possui elementos padronizados que devem ser seguidos: folha de rosto com as informações bibliográficas 
das patentes; relatório descritivo que indica o estado da técnica; reinvindicações que determinam o propósito da patente e seus elementos diferenciais; desenho, quando necessário, e resumo (TEIXEIRA; SOUZA, 2013; INPI, 2015). Segundo Antunes et al. (2012), a CIP é estruturada de forma hierárquica e contém seções, subseções, classes, subclasses, grupos e subgrupos que abrangem todas as áreas de tecnologia.

As bases de patentes apresentam facilidade de acesso às informações, bem como quantidade e qualidade dos trabalhos, facilitando a pesquisa e o desenvolvimento de novos produtos, processos e serviços (SILVA et al., 2016). Entretanto, depositar um pedido de patente não garante que esta seja concedida. Uma vez concedida, pode não ser licenciada ou explorada (PERUCCHI; MUELLER, 2014a). A partir do portal de periódicos da Coordenação de Aperfeiçoamento de Pessoal de Nível Superior (CAPES), é possivel acessar a base de dados da Derwent. Considerada fonte confiável para pesquisa de patentes mundiais, o banco de dados Derwent Patent Index (DWPI) é uma excelente fonte para coleta de indicadores de desenvolvimento, sendo abrangente em todo o mundo, com patentes aprimoradas. Possui pedidos e concessões de patentes e tem como fonte 44 autoridades mundiais que emitem tal certificação (RUAS; PEREIRA, 2014; MILANEZ, 2013).

\section{METODOLOGIA}

A fundamentação teórica apresentada foi baseada em pesquisa bibliográfica e o estudo é caracterizado como descritivo e exploratório de análise de depósitos de patentes. Para alcançar os objetivos propostos, foi realizado um levantamento mundial de patentes sobre a dengue e a influenza H1N1 utilizando a base de dados Derwent Innovations Index.

Para a pesquisa, foi utilizada a modalidade "Pesquisa Básica", com uso das expressões em língua inglesa com os delimitadores de tempo no período de 01 de janeiro de 2001 a 31 de dezembro de 2016. Foram construídos os seguintes indicadores: indicador de distribuição geográfica de países depositantes de patentes; indicador de tipologia de depositantes; indicador de classificação de patentes conforme a CIP; indicador de foco tecnológico.

No que concerne à análise dos dados, em conformidade com o estudo realizado por Almeida e Scatena (2016), estes foram analisados por técnicas descritivas (distribuição de frequência). A análise da evolução anual dos pedidos de patente foi realizada conforme estudo sobre análise de séries temporais em epidemiologia, realizado por Latorre e Cardoso (2001), sendo utilizado o processo de modelagem da série histórica iniciado pelo modelo de regressão linear simples e, em seguida, os modelos polinomiais de segundo e terceiro graus foram seguidos do exponencial.

\section{RESULTADOS E DISCUSSÃO}

Após revisão da literatura, coleta, processamento e análise dos dados levantados a partir da base Derwent Innovation Index, no período de janeiro de 2001 a dezembro de 2016, ao inserir a expressão "dengue", que inclui todos seus subtipos, foram encontradas 2.660 famílias de patentes, enquanto a expressão "H1N1" trouxe 1.103 famílias. A expressão "influenza" apresentou 13.511 documentos de patentes; e a expressão "influenza A", 9.872 documentos, na mesma base de dados e no mesmo período do estudo, ressaltando que o H1N1 é um subtipo da influenza A.

Os resultados encontrados corroboram a constatação da insuficiência de inovação em pesquisa e desenvolvimento quando o assunto é doença negligenciada ao ser comparada com qualquer outra enfermidade não negligenciada. Para ilustrar, segundo Feres e Silva (2016), ao realizar levantamento 
de patentes, em agosto de 2016, para algumas dessas doenças, na mesma base e no mesmo período deste estudo, foram encontrados: para Tuberculosis Pulmonary (Tuberculose Pulmonar) 2830 documentos de patentes; para Leprosy (Hanseníase) 2070 registros; Schistosomiasis (Esquistossomose) 862 registros enquanto que para a doença não negligenciada Myocardial Infarction (Infarto do Miocárdio) foram encontrados 18.774 registros de patentes.

Esses resultados mostram que as doenças negligenciadas apresentam 10\% da carga global das doenças e o câncer 5\%, sendo a categoria mais contemplada nas pesquisas e no setor farmacêutico. Diante disso, independentemente das taxas de morbimortalidade, o setor farmacêutico apresenta superioridade em inovar em relação a outros grupos de enfermidade que não as doenças negligenciadas (FERES; SILVA, 2016).

\section{DISTRIBUIÇÃO GEOGRÁFICA DOS PAIISESS DEPOSITANTES DE PATENTES}

A análise da distribuição geográfica foi realizada nos países onde foram realizadas as inscrições prioritárias, possibilitando obter informações sobre a concentração geográfica das doenças abordadas. Para a dengue, os Estados Unidos despontam em primeiro lugar com 50,26\% dos depósitos. Em seguida, encontra-se a Organização Europeia de Patentes, com 16,20\%, seguida da China, que conta com $8,46 \%$ dos depósitos. Logo após se encontra o Brasil, representando 4,14\% das inscrições prioritárias.

O país que apresentou maior número de depósitos com inscrição prioritária para H1N1, assim como para dengue, foram os Estados Unidos, com 32,64\%, destacando-se como o mais proeminente detentor das patentes analisadas. $O$ segundo maior país, a China, com 30,36\%, com uma diferença de apenas 2,28\% dos depósitos prioritários dos Estados Unidos. Diferentemente da análise para dengue, a República da Coréia encontra-se na terceira posição, com 11,88\% dos resultados encontrados. O Brasil, que figurava na $4^{a}$ posição para a dengue - com 4,14\% dos depósitos, para H1N1, encontra-se na $24^{a}$ posição, com apenas $0,18 \%$ dos depósitos.

Em consonância com os dados levantados nesta pesquisa, o trabalho realizado por Carvalho et al. (2015), que analisou documentos de patentes em Leishmaniose, doença também considerada negligenciada, entre 2008 e 2012, aponta que mesmo com a participação de outros países na tecnologia patentária, as instituições norte-americanas concentram geração significativa de tecnologias. Esse mesmo resultado também foi observado no trabalho de Almeida e Scatena (2016) sobre tuberculose pulmonar, com o destaque dos Estados Unidos, com $44.03 \%$ nas inscrições prioritárias de patentes, seguidos também da Organização Europeia de Patentes, com $16,61 \%$, da mesma forma que o encontrado para dengue neste trabalho.

\section{TIPOLOGIA DAS PATENTES SEGUNDO O DEPOSITANTE}

$\mathrm{Na}$ análise das patentes segundo o depositante, considerando tanto a dengue quanto $\mathrm{H} 1 \mathrm{~N} 1$, figurou com maior frequência a categoria Pessoa Física 33,65\% e 23,79\%, respectivamente. Para a dengue, a Empresa Farmacêutica 20,53\% apresentou-se na segunda colocação, seguida de Universidade com 12,28\%. Diferentemente dos dados já apresentados, para H1N1, a categoria Universidade 18,15\% apresentou a segunda maior frequência, enquanto Empresa Farmacêutica com 12,23\% figurou como quinta mais frequente, atrás de Instituto 15,19\% e Empresa Diversa 15,05\%.

Ao ser analisadas as famílias de patentes para dengue e H1N1, conforme seu depositante, verifica-se que a categoria pessoa física apresentou maior frequência 
em ambas as patologias, e é nessa categoria que estão os depositantes individuais que registram a invenção com o intuito de retorno futuro. Esse resultado corrobora os estudos supracitados, em que o pesquisador, aliado à Universidade ou à Empresa, desenvolve, cada vez mais, suas pesquisas. A natureza desses depositantes mostra qual o relacionamento entre as instituições e os pesquisadores. Houve pedidos de patentes com um único depositante, com vários titulares associados a universidades, empresas, institutos, entre outros.

As empresas, sejam elas farmacêuticas, de biotecnologia, ou mesmo de especialidades diversas, apresentaram-se de forma significativa no presente estudo. A categoria de Universidade apresentou-se como terceira mais frequente para dengue e como segunda mais frequente para H1N1. Como as universidades são pautadas na pesquisa, esperava-se que fosse obtida uma maior representatividade. Os dados levantados e discutidos anteriormente demonstram que, mesmo de forma insuficiente, são as empresas farmacêuticas, com os pesquisadores e as universidades, que estão apostando no desenvolvimento de novos produtos para as doenças em questão.

\section{CLASSIFICAÇÃO PREDOMINÁNTE DAS PATENTES CONFORME A CIP}

Internacionalmente usada, a classificação das patentes facilita a recuperação dos documentos, bem como a área do conhecimento abordada (MENDES et al., 2013). Todas as patentes apresentam uma classificação de acordo com a abordagem do seu conteúdo.

As classificações foram analisadas e posteriormente distribuídas conforme a classe, seguida da subclasse, de acordo com a Classificação Internacional de Patentes (CIP). Ao utilizar o descritor dengue, a Seção A (Necessidades Humanas) figurou com predominância $67,77 \%$, seguida da Seção
C (Química e Metalurgia) com 28,33\%. Da mesma maneira que para a dengue, ao utilizar o descritor H1N1, a Seção A predominou com a frequência de classificação seguida da Seção C, com respectivamente 61,00\% e $32,68 \%$ das categorias.

Foram encontradas para dengue e H1N1, respectivamente 34435 e 8272 classificações, visto que um documento de patente pode ter uma ou inúmeras classificações. Todas as patentes são classificadas de acordo com a aplicação e podem receber uma única classificação, ou várias, dependendo do tema abordado (MONTECCHI, 2013; SILVA et al., 2016).

O resultado de ambas as patologias mostrou uma maior frequência da classificação proveniente da seção A - Necessidades Humanas, que possui diversas subdivisões. A classe A61 (Ciência Médica ou Veterinária; Higiene) apresentou frequência predominante, sendo $63,64 \%$ para dengue e $57,65 \%$ para H1N1, do total das patentes classificadas sob os códigos das classes levantadas. A classe C07 (química orgânica) figurou como segunda mais frequente para dengue $(17,23 \%)$ e terceira mais frequente para H1N1 (12,52\%). Já a classe C12 (bioquímica; cerveja; álcool; vinho; vinagre; microbiologia; enzimologia; engenharia genética ou de mutação) foi a terceira mais frequente para dengue $(10,53 \%)$ e a segunda mais frequente para H1N1 (19,01\%). Essa análise permitiu identificar o resultado como bem-sucedido, uma vez que as classes de maior frequência estão diretamente relacionadas ao tema da pesquisa.

\section{FOCO TECNOLÓGICO}

A presente pesquisa mostrou que os principais focos das patentes levantadas são nas áreas de biotecnologia, farmacêutica, química orgânica e biologia em ambas as patologias. Tanto para dengue quanto para H1N1, a área de biotecnologia prevaleceu, com 40,19\% para dengue e 44,7\% para 
H1N1, seguida da área farmacêutica, que ocupou a segunda colocação, com 17,93\% para dengue e 19,9\% para H1N1. Houve algumas divergências entre as doenças. Para dengue, o terceiro foco tecnológico mais frequente foi Química Orgânica, com $17,67 \%$, enquanto que para ao H1N1, figurou a biologia, com $12,7 \%$.

Estudo realizado por Almeida e Scatena (2016), com abordagem em tuberculose pulmonar, diferentemente dos dados obtidos neste trabalho, obteve mais representatividade na área de química orgânica. Entretanto, em consonância com esta pesquisa, a área de farmacêutica figurou na segunda colocação. É importante ressaltar que, enquanto para o H1N1 obteve-se uma diversidade de 12 focos tecnológicos; para dengue, esse quantitativo passa para 15 focos, sendo que, além dos 12 apresentados para $\mathrm{H} 1 \mathrm{~N} 1$, ainda figurou tecnologia 0,04\%, eletrônicos 0,08\% e alimentos 0,19\%.

Segundo Rodrigues e Vieira (2016), a lucratividade e a competitividade existentes movem todo o mercado da indústria farmacêutica, exigindo altos investimentos, o que explica a concentração dessas indústrias nos países desenvolvidos. Além disso, é sabido o baixo retorno econômico do mercado quando as pesquisas são direcionadas para o desenvolvimento de drogas aos economicamente desprovidos.

\section{EVOLUÇÃO TEMPORAL DOS PEDIDOS DE PATENTES}

A evolução do número total de pedidos de patente de dengue no mundo, registrados na base de dados Derwent Innovation Index entre os anos de 2001 a 2016, foi ajustada por polinômio de $3^{\circ}$ grau, suavizada por média móvel centrada em três pontos e se apresentou crescente no período de 1996 a 2008, após isso, ocorreu decrescimento. A média no período estudado foi de 177,00 patentes, incremento de 22,43 ao ano e aceleração negativa de 1,40 ao ano, conforme apresentado na Figura 1. A tendência anual dos pedidos de patentes de H1N1 também foi ajustada por polinômio de $3^{\circ} \mathrm{grau}$ e se apresentou estável entre 1999 a 2004, crescente no período de 2004 a 2011. Após esse período, ocorreu decrescimento. A média no período estudado foi de 88,57 patentes, incremento de 21,19 ao ano e aceleração negativa de 1,02 ao ano, conforme ilustrado na Figura 2. Esse fato foi explicado pela pandemia de H1N1 em 2009, que atingiu todos os continentes e matou milhares de pessoas em todo o mundo. Esforços mundiais foram dispensados para controle da patologia, envolvendo pesquisas e inovação tecnológica (WHO, 2009).

O resultado da evolução anual dos pedidos de patente no período de 2001 a 2016 mostra um crescimento progressivo no número de registros de patentes para dengue com valor máximo em 2007, sendo o ano mais produtivo, com 428 registros, seguido de uma desaceleração nos anos seguintes. Para H1N1, o valor máximo no número de registro de patentes foi entre 2009 e 2011, com uma média de 173 registros ao ano. 


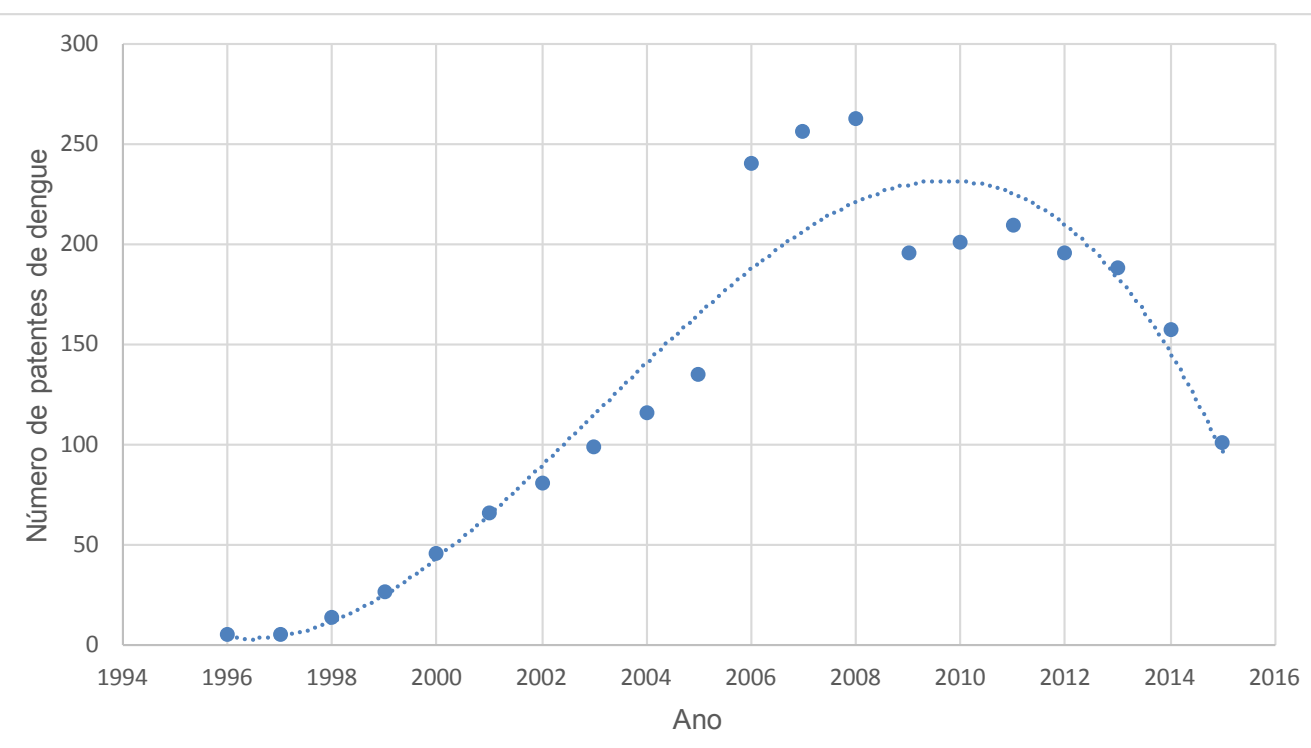

Figura 1 - Tendência anual dos pedidos de patentes de dengue no mundo, de 2001 a 2016 Fonte: Autoria própria (2017).

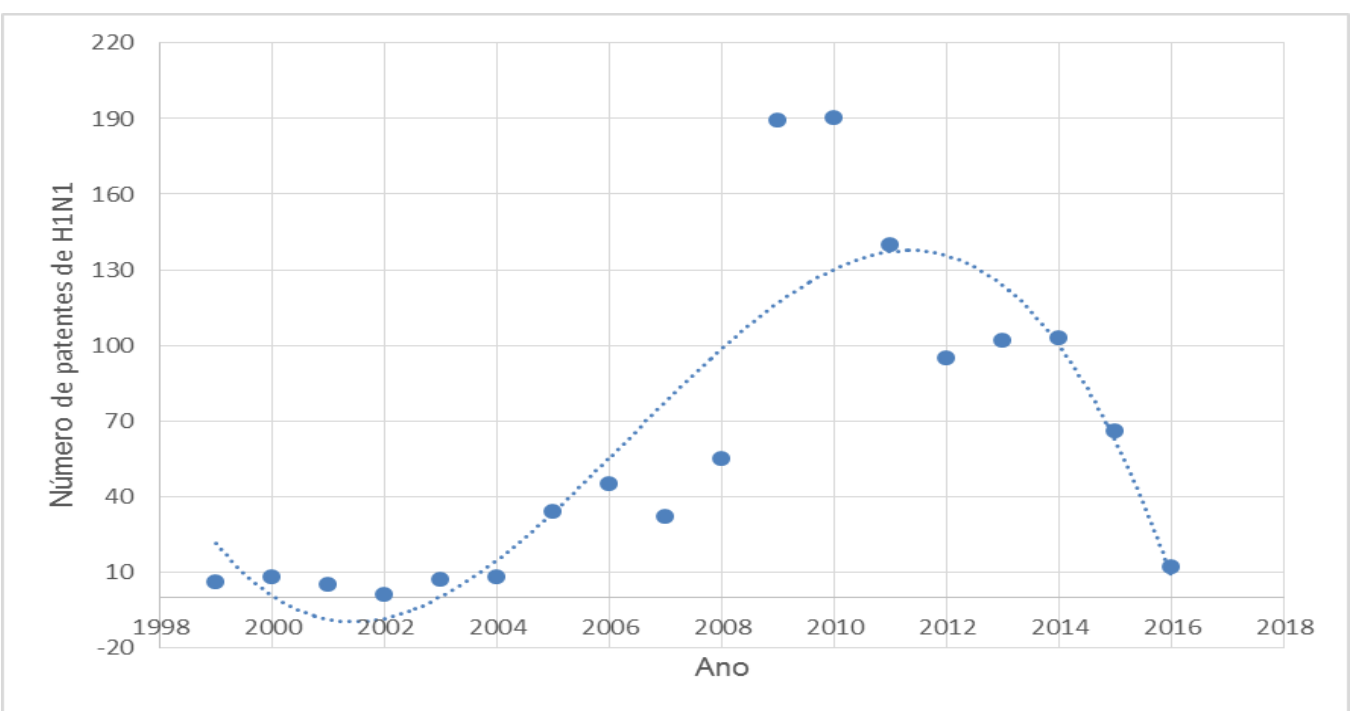

Figura 2 - Tendência anual dos pedidos de patentes de H1N1 no mundo, de 2001 a 2016 Fonte: Autoria própria (2017).

A desaceleração apresentada anteriormente no número de patentes ao ano ocorreu em diversas outras áreas, de forma geral, e em inúmeros escritórios do mundo, e pode ter sido devido à crise econômica global desencadeada em 2008, em especial, nos países da América do Norte e da Europa. Isso provocou uma diminuição nos investimentos oferecidos pelas empresas para pesquisa e desenvolvimento (ALMEIDA; SCATENA, 2016).

Conforme Teixeira e Souza (2013), a patente apresenta sigilo do conteúdo de até dezoito meses após o depósito. Isso pode ser considerada uma limitação da presente pesquisa, pois os dados referentes aos anos de 2015 e 2016 podem estar incompletos ao se pensar na demora em indexação nas bases correspondentes.

Importante ressaltar a disparidade encontrada entre o número de patentes e de artigos publicados para as doenças estudadas nesta pesquisa, sendo que Perucchi e Mueller (2014a) apontam que quando um pesquisador realiza a publicação de artigos, em revistas com boas classificações, a visibilidade e o reconhecimento são mais rapidamente observados. Caso a decisão 
seja por realizar um patenteamento, que garante propriedade ao inventor, ocorrerá um período mais longo de espera até que sejam realizadas todas as etapas do processo, necessitando sigilo e espera para publicação dos resultados na forma de artigo científico. Portanto, é importante que $\mathrm{o}$ pesquisador avalie as vantagens e desvantagens, em todos os sentidos, entre a publicação de seus trabalhos na forma de artigos científicos ou o registro destes como patente.

\section{CONCLUSÃO}

O avanço da ciência é estimulado pelo Sistema de Propriedade Intelectual. A exclusividade, obtida a partir das patentes, propicia a exploração comercial que, aplicada à saúde, possibilita a união de ciência e tecnologia voltadas para a saúde. As doenças negligenciadas, tais como a dengue, estão presentes principalmente nos países com desigualdades sociais, enquanto que a influenza A H1N1, hoje sazonal, pode ser encontrada em todos os continentes, desde a pandemia de 2009.

Diante desse cenário, foram realizados levantamento e análise dos pedidos de patentes para dengue e H1N1, no período entre 2001 a 2016, publicados na base de dados referencial de patentes Derwent Innovation Index (DII), para comparar o que tem sido feito no mundo relacionado a essas doenças. Além disso, os países em destaque, seus atores e principais características, podem ser úteis na identificação de lacunas e no direcionamento de futuras pesquisas na área.

O número de famílias de patentes encontrado para dengue foi de média a baixa representatividade, quando comparado a outras doenças não negligenciadas, resultado que pode ser justificado devido ao baixo interesse no desenvolvimento de pesquisas, mesmo com a importância epidemiológica da dengue para a população.
A distribuição geográfica demonstrou que as grandes potências econômicas são as detentoras do arcabouço patentário, com destaque norte-americano para ambas as patologias. Os números de documentos de patentes levantados retratam avanços, mas também a desigualdade presente entre diversos países do globo no quesito social, econômico, político ou tecnológico.

Ademais, a Classificação Internacional de Patentes permitiu verificar quais as tendências temáticas para ambas as patologias. Ao verificar os focos tecnológicos predominantes, tanto para dengue quanto para H1N1, foram elencados como mais frequentes as áreas de biotecnologia, farmacêutica, química orgânica e biologia.

Ao ser categorizados os depositantes, destacou-se como predominante o depósito de patentes por pessoa física para ambas as patologias. Esse resultado demonstra que o pesquisador, cada vez mais, busca apoio para o desenvolvimento das pesquisas aliando universidades e empresas. Enquanto para H1N1 grupos de pesquisa em âmbito mundial não medem esforços para a atualização anual da vacina, para os novos subtipos virais, decorrentes de conhecimento científico ampliado e consolidado, disponível para população há anos, como a dengue, ainda se necessita de muito empenho para tornar a vacina disponível para a população nas redes públicas de saúde, o que explica a colocação das empresas farmacêuticas frente a essa patologia.

Independentemente da estratégia adotada, conhecer o sistema de proteção de patentes é essencial para direcionar o processo de melhorias na saúde da população. Por todo o contexto deste estudo, é possível apontar as razões pelas quais existe tanta escassez de pesquisa para tratar doenças que acometem um número significativo da população. Espera-se, em médio prazo, que as inovações tecnológicas se tornem disponíveis para a população sob o risco das doenças estudadas nesta pesquisa. 


\section{AGRADECIMENTOS}

FAPEMIG, CAPES e CNPq 


\section{REFERÊNCIAS}

ALMEIDA, M. S.; SCATENA, L. M. Inovação tecnológica: um estudo sobre patentes em tuberculose pulmonar. 2016. 74f. Dissertação (Mestrado Profissional em Inovação Tecnológica) - Instituto de Ciências Tecnológicas e Exatas, UFTM, Uberaba, 2016.

Antunes, A. M. S. et al. Trends in Nanotechnology Patents Applied to the Health Sector. Recent Patents on Nanotechnology, v. 6, p. 29-43, 2012. Disponível em: https://pdfs.semanticscholar.org/ ec94/45c21f29f20a5298f02cfac3f82b3c78b792.pdf. Acesso em: 17 maio 2017.

BRASIL. Presidência da República. Lei $\mathbf{n}^{\circ} \mathbf{9 . 2 7 9}$, de 14 de maio de 1996. Regula os direitos e obrigações relativos à propriedade intelectual. Disponível em: http://www.planalto.gov.br/ccivil_03/leis/ 19279.htm. Acesso em: 20 ago. 2016.

BRASIL. Ministério da Saúde. Secretaria de Vigilância em Saúde. Monitoramento dos casos de dengue, febre de chikungunya e febre pelo vírus Zika até a Semana Epidemiológica 19, 2017 a.

Boletim Epidemiológico, Brasília, DF, v. 48, n. 16, 2017. Disponível em: http://portalarquivos.saude.gov.br/images/pdf/2017/maio/25/ Monitoramento-dos-casos-de-dengue-febre-de-chikungunya-efebre-pelo-virus-Zika-ate-a-Semana-Epidemiologica.pdf. Acesso em: 5 out. 2017.

BRASIL. Ministério da Saúde. Secretaria de Vigilância Sanitária. Influenza. Brasília, DF: Ministério da Saúde, 2017b. Disponível em: http://portalsaude.saude.gov.br/index.php/o-ministerio/principal/ secretarias/svs/influenza. Acesso em: 5 ago. 2017.

CALMANOVICl, C. E. A inovação, a competitividade e a projeção mundial das empresas brasileiras. Revista USP, São Paulo, n. 89, p. 190-203, 2011. Disponível em: file:///C:/Users/Note\%20-\%20W7/ Downloads/13877-16853-1-PB.pdf. Acesso em: 12 ago. 2017.

CARVALHO, C. L. C. et al. Panorama mundial de patentes publicadas entre 2008 e 2012 Com Foco Em Leishmaniose.

Cadernos de Prospecção, v. 8, n. 13, p. 459-468, jul./set. 2015. Disponivel em: https://portalseer.ufba.br/index.php/nit/article/ view/11777/pdf_7. Acesso em: 12 jul. 2017.

CARVALHO, M. S. Dengue: Teorias e Práticas. Caderno de Saúde Pública, Rio de Janeiro, v. 32, n. 4, 458p. maio. 2016. Disponível em: http://www.scielo.br/scielo.php?script=sci_arttext\&pid=S0102311X2016000401003. Acesso em: 10 set. 2016. 
DALBEM, A. G. et al. Dengue clássica e febre hemorrágica da dengue: etiologia, fisiologia, epidemiologia e fatores de risco.

Revista Ciência e Estudos Acadêmicos de Medicina. Cáceres, n. 1, p. 18-36, jan./jul. 2014. Disponível em: file:///C:/Users/Note\%20 -\%20W7/Downloads/60-162-1-PB.pdf. Acesso em: 19 set. 2017.

DESSOY, M. A. et al. Doenças tropicais negligenciadas: uma nova era de desafios e oportunidades. Química Nova, São Paulo, v. 36, n. 10, p. 1552-1556, 2013. Disponível em: http://www.producao.usp.br/ handle/BDPI/45204. Acesso em: 30 mar. 2016.

DIAS, L. B. A. et al. Dengue: transmissão, aspectos clínicos, diagnóstico e tratamento. Medicina, Ribeirão Preto, v. 43, n. 2, p. 143-151, 2010. Disponível em: http://www.revistas.usp.br/rmrp/ article/view/171/172. Acesso em: 12 abr. 2017.

FERES, M. V. C.; SILVA, A. R. A aspiração do sistema de patentes e o caso dos produtos terapêuticos para doenças negligenciadas. Revista Estudos Institucionais, v. 2, n. 2, p. 756-798, 2016. Disponível em: https://www.estudosinstitucionais.com/REl/article/ view/60/115. Acesso em: 10 ago. 2017.

Instituto Nacional da Propriedade Industrial (INPI). Manual para o depositante de patentes. Rio de Janeiro, 2015. Disponível em: http://www.inpi.gov.br/menu-servicos/patente/arquivos/manualpara-o-depositante-de-patentes.pdf. Acesso em: 10 ago. 2016.

LAMANA, S.; KOVALESKI, J. L. Patentes e o desenvolvimento econômico. In: CONGRESSO VIRTUAL BRASILEIRO DE ADMINISTRAÇÃO, 7., 2010. Anais eletrônicos [...] [S.I: s.n.], 2010. Disponível em: http://www.convibra.com.br/upload/paper/adm/ adm_1518.pdf. Acesso em: 1 nov. 2016.

LATORRE, M. R. D. O.; CARDOSO, M. R. A. Análise de séries temporais em epidemiologia: uma introdução sobre os aspectos metodológicos. Revista brasileira de epidemiologia, v. 4, n. 3, p. 145-152, 2001. Disponível em: http://www.scielosp.org/scielo. php?pid=S1415-790X2001000300002\&script=sci_abstract\&tlng=pt. Acesso em: 15 fev. 2016.

LINDOSO, J. A.; LINDOSO, A. A. B. P. Neglected tropical diseases in Brazil. Revista do Instituto de Medicina Tropical de São Paulo, São Paulo, v. 5, n. 51, p. 247-253, 2009. Disponível em: http://www.scielo. $\mathrm{br} / \mathrm{scielo}$.php?script=sci_arttext\&pid=S0036-46652009000500003. Acesso em: 11 abr. 2017. 
LINDOSO, J. A. et al. Visceral Leishmaniasis and HIV Coinfection in Latin America. PloS Neglected Tropical Diseases, San Francisco, set. 2014. Disponível em: http://journals.plos.org/plosntds/ article?id=10.1371/journal.pntd.0003136. Acesso em: 20 nov. 2016.

LUNA, F. et al. Impacto das marcas e patentes no desempenho econômico das firmas. In: ENCONTRO NACIONAL DE ECONOMIA, 35., 2007, Niterói, RJ. Anais eletrônicos [...] Niterói, RJ: ANPEC, 2007. Disponível em: http://econpapers.repec.org/paper/ anpen2007/155.htm. Acesso em: 28 set. 2016.

MANIERO, V. C. et al. Dengue, chikungunya e zika vírus no brasil: situação epidemiológica, aspectos clínicos e medidas preventivas. Almanaque multidisciplinar de pesquisa, Rio de Janeiro, v. 1, n. 1 , p. 118-147, 2016. Disponível em: http://publicacoes.unigranrio.edu. br/index.php/amp/article/view/3409/2114. Acesso em: 23 jan. 2017.

MENDES, L. et al. Patent applications on representative sectors of biotechnology in Brazil: an analysis of the last decade. Journal of Technology Management \& Innovation, Santiago, v. 8, n. 4, p. 91-102, 2013. Disponível em http://www.jotmi.org/index.php/GT/ article/view/1392/881. Acesso em: 17 nov. 2016.

MILANEZ, D. H. et al. Assessing nanocellulose developments using science and technology indicators. Material Research, São Carlos, v. 16, n. 3, p. 635-641, jun. 2013. Disponível em: http://www.scielo. $\mathrm{br} / \mathrm{scielo}$. php?script=sci_arttext\&pid=S1516-14392013000300014. Acesso em: 18 nov. 2017.

MONTECCHI, T.; RUSSO, D.; Liu, Y. Searching in cooperative patent classification: comparison between keyword and concept-based search. Advanced Engineering Informatics, v. 27, n. 3, p. 335-345, ago. 2013. Disponível em: http://www.sciencedirect.com/science/ article/pii/S1474034613000219. Acesso em: 14 jun. 2016.

MOREL, C. M. Inovação em saúde e doenças negligenciadas.

Cadernos de Saúde Pública, Rio de Janeiro, v. 22, n. 8, p. 15221523, ago. 2006. Disponível em: http://www.scielo.br/scielo. php?script=sci_arttext\&pid=S0102-311X2006000800001. Acesso em: 6 jul. 2016.

PAULA, M. F.; RIBAS, J. L. C. A epidemiologia da influenza a (H1N1). Caderno Saúde e Desenvolvimento, v. 7, n.4, p. 42-45, jan./jun. 2015. Disponível em: https://www.uninter.com/cadernosuninter/ index.php/saude-e-desenvolvimento/article/view/423/350. Acesso em: 5 dez. 2016. 
PEREIRA, J. M. A gestão do sistema de proteção à propriedade intelectual no Brasil é consistente? Revista de Administração Pública, Rio de Janeiro, v. 45, n. 3, p. 567-590, maio/jun. 2011. Disponível em: http://www.scielo.br/scielo.php?pid=S0034$76122011000300002 \&$ script=sci_abstract\&tIng=PT. Acesso em: 6 abr. 2016.

PEREIRA, A. I. S. et al. Atividade antimicrobiana no combate as larvas do mosquito Aedes aegypti: homogeneização dos óleos essenciais do linalol e eugenol. Educación Química, México, v. 25, n. 4, p. 446449, 2014. Disponível em: http://www.sciencedirect.com/science/ article/pii/S0187893X14700655. Acesso em: 19 jun. 2016.

Perucchi, V.; Mueller, S. P. M. Estudo com as patentes produzidas e o perfil dos inventores dos Institutos Federais de Educação, Ciência e Tecnologia. Revista Digital de Biblioteconomia e Ciência da Informação, Campinas, v. 12, n. 1, p. 191-213, jan./abr. 2014a. Disponível em: https://periodicos.sbu.unicamp.br/ojs/index.php/ rdbci/article/view/1624/pdf_55. Acesso em: 10 maio 2016.

Perucchi, V.; Mueller, S. P. M. Universidades e a produção de patentes: tópicos de interesse para o estudioso da informação tecnológica. Perspectivas em ciência da informação, Belo Horizonte, v. 19, n. 2, p. 15-36, abr./jun. 2014b. Disponível em: http://portaldeperiodicos.eci.ufmg.br/index.php/pci/article/ view/1828/1392. Acesso em: 29 maio 2016.

RODRIGUES, C. R. Análise do registro de patentes para doenças negligenciadas no Brasil (1996-2013): prioridades e necessidades do SUS. 2013. 75f. Trabalho de Conclusão de Curso (Monografia) - Faculdade de Ceilândia, Universidade de Brasília, 2013.

Disponível em: http://bdm.unb.br/bitstream/10483/7705/1/2013_ CleversonRubensRodrigues.pdf. Acesso em: 4 abr. 2016.

ROSSETTO, E. V.; LUNA, J. A. Relacionamento entre bases de dados para vigilância da pandemia de influenza $A(H 1 N 1)$ pdm09, Brasil, 2009-2010. Adero de Saúde. Caderno de Saúde Pública, Rio de Janeiro, v. 32, n. 7, jul. 2016. Disponível em: http://www. scielo.br/scielo.php?pid=S0102-311X2016000705002\&script=sci_ abstract\&tlng=pt. Acesso em: 10 jul. 2017.

RODRIGUES, A. B.; VIEIRA, R. S. A tecnologia na indústria farmacêutica e as doenças negligenciadas: uma análise preliminar sobre o surto do vírus ebola. Espacios, v. 37, n. 4, nov. 2016. Disponível em: http://www.revistaespacios.com/ a16v37n04/16370411.html. Acesso em: 19 jul. 2017. 
RUAS, T. L.; PEREIRA, L. Como construir indicadores de Ciência, Tecnologia e Inovação usando Web of Science, Derwent World Patent Index, Bibexcel e Pajek? Perspectivas em Ciência da Informação, Belo Horizonte, v. 19, n. 3, p. 52-81, set. 2014. Disponível em: http://www.scielo.br/scielo.php?pid=S1413$99362014000300004 \&$ script=sci_abstract\&tlng=pt. Acesso em: 13 jul. 2016.

SHARP, T. M. et al. A New Look at an Old Disease: Recent Insights into the Global Epidemiology of Dengue. Current Epidemiology Reports, v. 4, n. 1, p. 11-21, jan. 2017. Disponível em: https://www. ncbi.nlm.nih.gov/pmc/articles/PMC5306284/. Acesso em: 18 ago. 2017.

SILVA, J. N. et al. Estudo prospectivo sobre propriedades antineoplásicas de plantas da família Fabaceae com ênfase em Mimosa caesalpiniifolia. Geintec, São Cristóvao, v. 6, n. 3, p. 3304-3318, 2016. Disponível em: https://www.researchgate.net/ publication/308926671_Estudo_prospectivo_sobre_propriedades_ antineoplasicas_de_plantas_da_familia_Fabaceae_com_enfase_em_ Mimosa_caesalpiniifolia_Benth. Acesso em: 19 fev. 2016.

SOUZA, W. S. Doenças Negligenciadas: Ciência e tecnologia para o desenvolvimento Nacional- Estudos estratégicos. Rio de Janeiro: Academia Brasileira de Ciências, 2010. 56p. Disponível em: https:// www.abc.org.br/IMG/pdf/doc-199.pdf. Acesso em: 12 fev. 2016.

Teixeira, R. C.; Souza, R. R. O uso das informações contidas em documentos de patentes nas práticas de Inteligência Competitiva: apresentação de um estudo das patentes da UFMG. Perspectivas em Ciência da Informação, Belo Horizonte, v. 18, n. 1, p. 106-125, jan./mar. 2013. Disponível em: http://portaldeperiodicos.eci.ufmg.br/ index.php/pci/article/view/844/1108. Acesso em: 20 ago. 2017.

VALLE, D. et al. Zika, dengue e chikungunya: desafios e questões. Epidemiologia e serviços de saúde, Brasília, v. 25, n. 2, p. 419422, abr./jun. 2016. Disponível em: http://www.scielo.br/pdf/ress/ v25n2/2237-9622-ress-25-02-00419.pdf. Acesso em: 10 mar. 2017.

World Health Organization (who). Pandemic (H1N1) 2009 - update 60. Geneve, 2009. Disponível em: http://www.who.int/csr/don/ h1n1_20090731_weekly.png?ua=1http://www.who.int/csr/disease/ swineflu/notes/briefing_20100810/en/. Acesso em: 11 abr. 2017.

World Health Organization (who). Influenza. Geneve, 2017a. Disponível em: Disponível em: http://www.who.int/influenza/en. Acesso em: 13 set. 2017. 
World Health Organization (WHO). Influenza: vaccines. Geneve, 2017b. Disponível em: http://www.who.int/influenza/vaccines/en/. Acesso em: 10 ago. 2017. 